\title{
Robust Data Transmission Rate Allocation to Improve Energy Efficiency in 6G Networks
}

\author{
Azadeh Pourkabirian \\ Faculty of Computer and Information Technology Engineering \\ Qazvin Branch, Islamic Azad University \\ Qazvin, Iran \\ pourkabirian@qiau.ac.ir
}

\author{
Mohammad Hossein Anisi \\ School of Computer Science and Electronic Engineering \\ University of Essex \\ Colchester, U.K \\ m.anisi@essex.ac.uk
}

\begin{abstract}
The future sixth-generation (6G) network is expected to support both sensing and communications. Since the sensing performance will highly rely on the residual battery of smart devices, energy efficiency is one of the main concerns in the design of 6G. Motivated by these facts, we design an energy efficient data transmission rate allocation approach for $6 \mathrm{G}$ networks. To have a more realistic deployment, we assume that perfect channel state information is not available. Imperfect channel state information (CSI) might waste energy sources or degrade quality of service (QoS). Thus, we apply the maximum likelihood estimation (MLE) method to estimate the true channel characteristics for a given set of observations. The proposed approach is robust against unknown channel statistics and allows adapting UEs transmission rate to the channel quality which reduce energy consumption and guarantees QoS. Both numerical analysis and simulation results confirm the effectiveness of the proposed work in terms of energy efficiency and throughput maximization.
\end{abstract}

Keywords- energy efficiency, maximum likelihood estimation, data transmission rate allocation, $6 G$ networks

\section{INTRODUCTION}

Recently, 6G wireless communication network has drawn considerable attention both from academia and industry [1][4]. $6 \mathrm{G}$ seems to be a promising technology to overcome the major constraints of the previous generations of cellular network. Some of the motivating trends [5][6] of the 6G development include: higher date rate, higher spectral efficiency, higher energy efficiency, lower latency, higher reliability and more QoS guarantees. However, the performance of $6 \mathrm{G}$ network will depend on the available energy at limited battery life devices such as sensors and smartphones which are tightly integrated in this autonomous network. Although integration of battery-free smart devices and wireless information and energy transfer (WIET) is a new enabling technology for lowering the energy consumption however, energy efficiency in 6G network is still a critical issue. According to studies [7], every year, the information and communications technology (ICT) systems which consume energy sources for communication and data transmission increase the global world $\mathrm{CO} 2$ emissions by $5 \%$. This amount will rapidly increase with the advent of $6 \mathrm{G}$ networks comprising of billions of connected devices in the near future. Therefore, an energy efficient power optimization approach needs to minimize overall energy consumption. Power allocation strategies are efficient techniques for energy efficiency enhancement in wireless communication networks. Many power optimization solutions were developed in the literature. Authors in [8] established a resource allocation method for multiple access edge computing (MEC)-enabled heterogeneous networks (HetNets) that reduces the energy consumption for power-limited wireless devices. Authors in [9] developed a machine learning-based mobility management scheme for $6 \mathrm{G}$ networks. They also proposed a 6G-based network in box (NIB) framework with high energy efficiency that guarantees users' QoS, and QoE in 6G-based industrial NIB. Study [10] investigated a QoS-based energy optimization method for IoT devices in $6 \mathrm{G}$ networks. The authors also established a 6G-based multimedia data structure framework to evaluate QoE with acquisition time for energy-efficient transmissions. A successive interference cancellation (SIC) approach for multi-carrier multi-user $6 \mathrm{G}$ networks was investigated in [11]. The authors proposed a joint user association, SIC ordering and subcarrier assignment method to improve the worst-case network energy efficiency. The energyefficient power optimization problem for wireless communication was addressed in [12]. The authors proposed a monotonic lower-complexity framework based on sequential programming which obtains suboptimal transmit power strategies. They claimed that the proposed framework achieves global optimality transmit power for practical scenarios. Authors in [13] proposed a robust channel estimation method which optimizes energy consumption in downlink 5G communications. They modeled the optimization problem as a non-cooperative game to achieve the best energy consumption strategy under the worst-case channel uncertainty. An energy minimization problem was addressed for industrial 6G applications in [14]. Authors applied distributed artificial intelligence (DAI) to cluster the wireless devices for efficiently resource allocation and then used the back propagation neural network (BPNN) and convolutional neural network (CNN) for energy optimization. Study [15] analyzes energy efficiency optimization problem for multiple-input-single-output (MISO) communications. The authors used zero-forcing beamforming method to improve the network energy efficiency under power 
and energy consumption constraints. The authors in [16] developed dynamic resource allocation using the VickreyClarke-Groves auction game theory. They also consider both cross-tier interference and users' QoS requirements in the resource allocation process.

In this paper, we propose a robust data transmission rate approach in order to enhance energy efficiency in 6G network. As we know, optimal rate allocation requires exact information of channel parameters. On the other hand, we assume that the perfect channel state information is not available that is a more realistic scenario for wireless communications. Thus, we use maximum likelihood estimation method in order to obtain accurate information of channel characteristics. The proposed robust estimation approach minimizes the estimation error and obtains the accurate values of the channel parameters. We also design a distributed algorithm to achieve the solution of the optimization problem. In the proposed algorithm, each UE obtains true channel coefficients and then adjusts the optimal transmission power level. Then, the optimal transmission rate allocation strategy is derived as a solution of the problem. The performance evaluation prove the superiority of the proposed work in terms of high energy efficiency and throughput.

The rest of this paper is organized as follows. Section II introduces the system description and problem formulation.. Section III provides the simulation analysis and performance evaluation of the proposed approach. Finally, the main conclusions are discussed in Section IV.

\section{PROBLEM DESCRIPTION}

\section{A. System Model}

Consider a multiuser 6G network where there exists $M$ base stations (BSs), each equipped with $N_{B S}$ antennas and $K$ wireless user equipment (UE) with $N_{U E}$ antennas. We assume that BSs and UEs are located based on two independent homogeneous Poisson point process (PPP) with intensity $\lambda_{B}$ and $\lambda_{U}$, respectively.

Definition 1. Energy efficiency can be defined [17] as the ratio of bit rate to the total power consumption $\left(E_{\text {eff }}[\right.$ bit $/ J$ Joule $\left.]=\frac{\text { bit rate }(\text { bit } / s)}{\text { power consumption }}\right)$. Thus, the total energy efficiency of a wireless multiuser system can be expressed as:

$$
E_{e f f}=\frac{\sum_{i=1}^{K}\left(\frac{W}{K}\right) \log _{2}\left(1+\frac{P_{i}^{t r}\left|g_{i j}^{H} h_{i}\right|^{2}}{I_{i}+n_{i}}\right)}{\sum_{i=}^{K} P_{i}^{\text {Con }}}
$$

Where $W$ denotes the total network bandwidth, $P_{i}^{t r} \in P^{t r}$ is the transmission power of UE $i$ in which $P^{t r} \in \mathbb{R}^{\mathrm{K} \times N_{U E}}, g_{i j}^{H}$ identifies equalizing gain for UE $i$ from $B S_{j}, h_{i}=$ $\left[h_{i, 1}, h_{i, 2}, \ldots, h_{i, N_{U E}}\right]^{T}, h_{i} \in H$ denotes the fading channel coefficients between UE $i$ and its serving BS in which $H \in \mathbb{R}^{\mathrm{K} \times N_{U E}}$ describes Rayleigh fading channel, $I_{i} \approx$ $\sum_{k \neq i}\left(P_{k}^{t r}\left|g_{k i}^{H} h_{k}\right|^{2}\right)$ is the received aggregate interference from all interfering users, $n_{i}$ is the white Gaussian noise with $N \sim(0, \mathbf{I})$ and $P_{i}^{c o n}$ states the total power consumption of UE $i$.
For the sake of simplicity, we substitute $\left(\frac{W}{K}\right) \log _{2}(1+$ $\left.\frac{P_{i}^{t r}\left|g_{i j}^{H} h_{i}\right|^{2}}{I_{i}+n_{i}}\right)$ with $R_{i}\left(h_{i}\right)$ as the data transmission rate of UE $i$ in the rest of the paper. The main objective is to maximize the network energy efficiency (i.e., $\max _{P_{i}^{t r}} \int_{t=0}^{T} E_{\text {eff }}(t)$ ).

Remark 1. According to (1), energy efficiency is maximized if $R(H) / p_{\text {con }} \rightarrow \infty$. Thus, taking the power consumption $P^{\text {con }} \rightarrow 0$, the data transmission rate $R(H) \rightarrow \infty$, or a combination thereof achieves the energy efficiency maximization. However, Energy efficiency maximization does not always mean minimizing the power consumption. Energy efficiency can be maximized by increasing data transmission rates. Thus, the optimization problem can be expressed as below:

$$
\begin{aligned}
& \max _{P^{t r}, H} E_{\text {eff }}(R(H))=\max _{P^{t r}, H^{H}} R\left(\left(P_{1}^{t r}, \ldots, P_{K}^{t r}\right) ; H^{H}\right) \\
& \text { Subject to: } C_{1}: 0<P_{i}^{t r} \leq P_{\max } \\
& C_{2}: R_{i}\left(h_{i}\right) \geq R_{\text {min }}, i=1, \ldots, K \\
& C_{3}: \sum_{i=1}^{K} \operatorname{Tr}\left(\Sigma_{h_{i}}\right) \leq \varepsilon \\
& C_{4}: \Sigma_{h_{i}} \geq 0
\end{aligned}
$$

Where $R\left(\left(P_{1}^{t r}, \ldots, P_{K}^{t r}\right) ; H^{H}\right)=$ $\bigcup_{\left\{\Sigma_{P_{i} \geq 0, T r}\left(\Sigma_{P_{i}}\right) \leq P_{\max }, \forall i\right\}}\left\{\left(R_{1}, \ldots, R_{K}\right): \sum_{i \in K} R_{i} \leq \log \mid I+\right.$ $\left.\left.\sum_{i \in K} h_{i}^{H} \Sigma_{P_{i}} h_{i} \mid\right\},\right), R_{\text {min }}$ is the minimum data transmission rate for QoS guarantees, $\Sigma_{P_{i}}=P_{i}^{t r}\left(P_{i}^{t r}\right)^{H}$ and $\Sigma_{h_{i}}=$ $h_{i}\left(h_{i}\right)^{H}$ identify the covariance matrices of transmission power and channel coefficients, respectively. It is obvious from remark1 that the data transmission rate strongly depends on channel characteristics (i.e., $H^{H}$ ). On the other hand, the optimal data transmission rate can be allocated under known channel conditions. In the absent of perfect knowledge of channel state, we estimate the unknown parameters of channel (i.e., $\widehat{H}=H+$ $\left.\Delta_{H}\right)$ using MLE method where $\Delta_{H} \sim N\left(0, \xi^{2} \mathbf{I}\right)$ identifies the estimation error and $\xi^{2}$ states the error variance. Note that in estimation process, the objective is minimizing the estimation error $\left(\left\|\Delta_{H}\right\|_{2}=\|\widehat{H}-H\|_{2} \leq \varepsilon\right)$. Therefore, the above optimization problem can be reformulated as below:

$$
\max _{P^{t r}, H} E_{e f f}\left(\Sigma_{P}, \widehat{H}\right)
$$

$$
\text { Subject to: } \operatorname{Tr}\left((\widehat{H}-\Delta) \Sigma_{P}(\widehat{H}-\Delta)^{H}\right) \leq P_{\max } \boldsymbol{I}
$$

Thus, the optimal data transmission rate can be obtained by the solution of the optimization problem in (3) as below:

$$
\widehat{H}=\arg \max _{P^{t r}, H} E_{e f f}\left(\Sigma_{P}, R(\widehat{H})\right)
$$


Let $f_{i}\left(y_{i} \mid \hat{h}_{i}\right)$ be as the probability density function where $y_{i}\left(h_{i}\right)=F h_{i} \sqrt{P^{t r}} S+\omega$ denotes the observed signal at UE $i, F$ states the precoding matrix, $s \in \mathbb{R}^{N_{U E} \times 1}$ states the transmitted symbols vector and $\omega \in \mathbb{R}^{N_{U E} \times 1}$ indicates Gaussian observation noise vector. As we know, MLE method obtains the perfect value of the unknown parameter through maximizing probability density on the observed data. Our goal is to find the optimal $\hat{h}_{i}^{o p t}$ (i.e., $\left\|\hat{h}_{i}^{o p t}-h_{i}\right\|_{2} \leq \epsilon, \forall i \in K$ ) that maximizes the probability density of the observed signal $y_{i}$. Here, we define the likelihood function as follows:

$$
L_{i}\left(\hat{h}_{i} \mid y_{i}\right)=f_{i}\left(y_{i} \mid \hat{h}_{i}\right)
$$

Without loss of generality, consider $y_{i} \in\left[y_{i j}, y_{i j}+\delta\right], j=$ $1, \ldots, N_{U E}$ in which $\delta>0$. Therefore, we have:

$$
\arg \max _{\widehat{h}_{i}} L_{i}\left(\hat{h}_{i} \mid y_{i}\right)=\arg \max _{\widehat{h}_{i}} \frac{1}{\delta} L_{i}\left(\hat{h}_{i} \mid y_{i} \in\left[y_{i j}, y_{i j}+\delta\right]\right)
$$

Consider $\delta$ is a constant. We can state that:

$$
\begin{aligned}
& \arg \max _{\hat{h}_{i}} \frac{1}{\delta} L_{i}\left(\hat{h}_{i} \mid y_{i} \in\left[y_{i j}, y_{i j}+\delta\right]\right)= \\
& \arg \max _{\hat{h}_{i}} \frac{1}{\delta} \operatorname{Pr}\left(y_{i j} \leq y_{i} \leq y_{i j}+\delta \mid \hat{h}_{i}\right)= \\
& \arg \max _{\hat{h}_{i}} \frac{1}{\delta} \int_{y_{i j}}^{y_{i j}+\delta} f_{i}\left(y_{i} \mid \hat{h}_{i}\right) d y_{i}
\end{aligned}
$$

Then

$$
\begin{aligned}
& \arg \max _{\hat{h}_{i}} L_{i}\left(\hat{h}_{i} \mid y_{i} \in\left[y_{i j}, y_{i j}+\delta\right]\right)= \\
& \arg \max _{\hat{h}_{i}} \frac{1}{\delta} \int_{y_{i j}}^{y_{i j}+\delta} f_{i}\left(y_{i} \mid \hat{h}_{i}\right) d y_{i}
\end{aligned}
$$

Using L'Hôpital's rule, the following results can be explicitly expressed:

$$
\begin{aligned}
& \lim _{\delta \rightarrow 0^{+}} \frac{1}{\delta} \int_{y_{i j}}^{y_{i j}+\delta} f_{i}\left(y_{i} \mid \hat{h}_{i}\right) d y_{i}=\lim _{\delta \rightarrow 0^{+}} \frac{\frac{d}{d \delta} \int_{y_{i j}}^{y_{i j}+\delta} f_{i}\left(y_{i} \mid \widehat{h}_{i}\right) d y_{i}}{\frac{d \delta}{d \delta}}= \\
& \lim _{\delta \rightarrow 0^{+}} \frac{f\left(y_{i j}+\delta \mid \widehat{h}_{i}\right)}{1}=f\left(y_{i j} \mid \hat{h}_{i}\right)
\end{aligned}
$$

And

$$
\begin{gathered}
\arg \max _{\hat{h}_{i}} L_{i}\left(\hat{h}_{i} \mid y_{i j}\right)=\arg \max _{\hat{h}_{i}}\left[\operatorname { l i m } _ { \delta \rightarrow 0 ^ { + } } L _ { i } \left(\hat{h}_{i} \mid y_{i} \in\right.\right. \\
\left.\left.\left[y_{i j}, y_{i j}+\delta\right]\right)\right]=\arg \max _{\hat{h}_{i}}\left[\lim _{\delta \rightarrow 0^{+}} \frac{1}{\delta} \int_{y_{i j}}^{y_{i j}+\delta} f_{i}\left(y_{i} \mid \hat{h}_{i}\right) d y_{i}\right]= \\
\arg \max _{\hat{h}_{i}} f\left(y_{i j} \mid \hat{h}_{i}\right)
\end{gathered}
$$

Hence, it can be said that

$$
\hat{h}_{i}^{o p t}=\arg \max _{\widehat{h}_{i}} L_{i}\left(\hat{h}_{i} \mid y_{i j}\right)=\arg \max _{\widehat{h}_{i}} f\left(y_{i j} \mid \hat{h}_{i}\right)
$$

For the sake of simplicity, we use $\hat{h}$ instead of $\hat{h}_{i}$. Then we calculate $\hat{h}$ for a typical UE as follows:

$$
M(\hat{h})=\left[\begin{array}{ccc}
\left.\frac{\partial^{2} L}{\partial h_{1}^{2}}\right|_{h=\widehat{h}} & \cdots & \left.\frac{\partial^{2} L}{\partial h_{1} \partial h_{N_{U E}}}\right|_{h=\widehat{h}} \\
\vdots & \ddots & \vdots \\
\left.\frac{\partial^{2} L}{\partial h_{N_{U E}} \partial h_{1}}\right|_{h=\widehat{h}} & \cdots & \left.\frac{\partial^{2} L}{\partial h_{N_{U E}}^{2}}\right|_{h=\widehat{h}}
\end{array}\right]
$$

Where $M(\hat{h})$ is Hessian matrix.

Lemma1. For independent and identically distribution observations (i.i.d), the proposed estimator $(L(\hat{h} \mid y))$ uniformly converges to $L(h)$ on $Y$ as:

$$
\sup _{h \in H}|L(\hat{h} \mid y)-L(h)| \stackrel{p}{\rightarrow} 0, \forall y \in Y
$$

Proof. According to the uniform law of large numbers, there exists the pointwise convergence since the following conditions are fulfilled:

- $H$ is compact.

- $\forall h \in H$ and $\forall y \in Y, f(y, h)$ is continuous.

- There is a dominating function $g(y)$ where $\mathbb{E}[g(y)]<$ $\infty$, and $\forall h \in H,\|f(y, h)\| \leq g(y)$.

Clearly, $\mathbb{E}[g(y)]$ is continuous in $h$. Therefore, the following can be expressed:

$$
\sup _{h \in H}\left\|\frac{1}{K} \sum_{i=1}^{K} f\left(y_{i}, h\right)-\mathbb{E}[f(y, h)]\right\| \stackrel{a . s}{\rightarrow} 0
$$

On the other hand, $L(\hat{h} \mid y)$ is uniform convergence on $\mathrm{Y}$ if $\forall h \in H$ and $\forall y \in Y, \exists \varepsilon>0,\left|f_{y}(\hat{h})-f(h)\right|<\varepsilon$. In other word, unif $\lim _{y \rightarrow \infty} f_{y}=f$.

Without loss of generality, we can express

$$
g_{y}=\sup _{h \in H}|L(\hat{h} \mid y)-L(h)|=\sup _{y \in Y}\left|f_{y}(\hat{h})-f(h)\right|
$$

Thus, it can be said that $f_{y}(\hat{h})$ uniformly converges to $f(h)$ if and only if $g_{y} \rightarrow 0$ when $y \rightarrow \infty$.

\section{B. Data Transmission Rate Allocation Algorithm}

Here, we introduce a distributed transmission rate allocation algorithm. In this algorithm, each UE estimate optimal channel coefficients and then adjust its transmission power to the channel quality. The UE calculate the received payoff in terms of data transmission rate per the total power consumption. UEs change their transmission power level if achievable transmission rate is less than the minimum UE transmission rate for QoS guarantees. The UE then updates the current channel state. Table I provides the pseudo-code for the energy efficient data transmission rate approach. 
TABLE I. TRANSMisSion RATE ALlOCATION AlgorithM

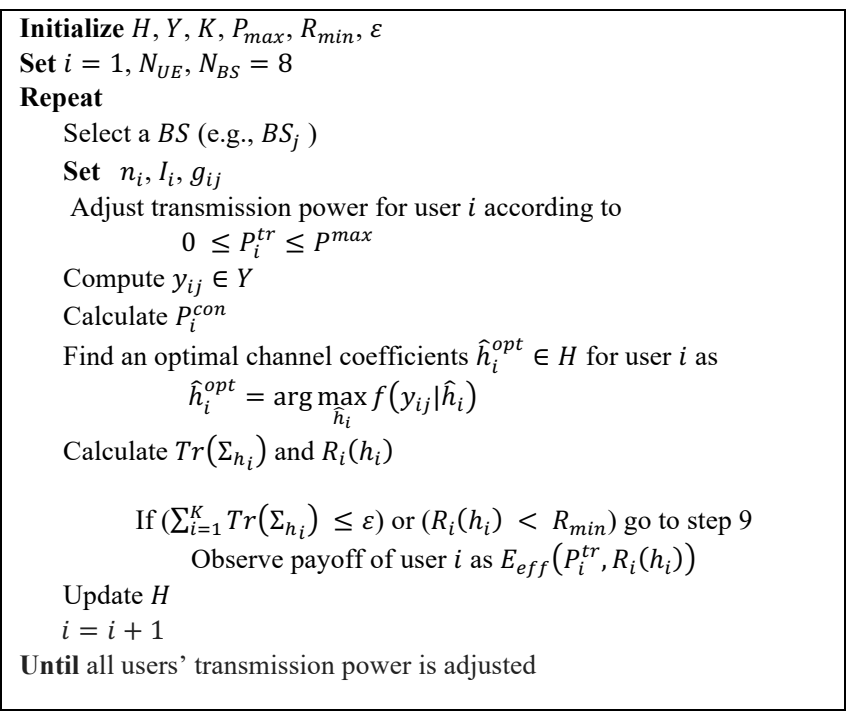

\section{Simulation RESUlts}

In this section, we provide the performance comparison of our proposed method with a ML-based energy-efficient mechanism in [9] and SIC ordering energy-efficient resource management scheme in [11]. The evaluation results were performed by the CVX package in MATLAB and averaged over 1200 run. Simulation results were obtained based on geometric programming (GP) mode of CVX which accepts all the special functions and transforms them into convex form. CVX handles the problem directly, since the norms are part of the base library of it. We consider a multiuser $6 \mathrm{G}$ network consisting of 9 BSs that each is equipped with $N_{B S}=8$ antennas and 60 UEs with $N_{U E}=4$ antennas. Both BSs and UEs are distributed randomly in a $1600 \mathrm{~m} \times 1600 \mathrm{~m}$ area. We set the transmission power of BSs to $45 \mathrm{dBm}$ and initialize UEs' transmission power based on the distance to their serving BSs in the range of $7 \mathrm{dBm}$ to $30 \mathrm{dBm}$. Without loss of generality, we also set $\xi^{2}=0.01$ as the identical error variance, $\sigma_{n}^{2}=0.01, P_{\max }=35 \mathrm{dBm}$ and $P_{\text {min }}=7$ $\mathrm{dBm}$. The fading channel is modeled according to Rayleigh statistic and the channel matrix is obtained by averaging 800 random channel realizations.

Fig. 1 plots the impact of variation of transmission power on the energy efficiency of the network under different number of wireless users. We vary the transmission power of UEs from $5 \mathrm{dBm}$ to $35 \mathrm{dBm}$ and change the number of users from 15 to 60 . We observe that the energy efficiency first gives rise as the transmission power increases, then it is saturated when the transmission power reaches $35 \mathrm{dBm}$. The major reason behind the results is that our method adjusts the optimal transmission power based on the accurate CSI obtained by maximum likelihood estimator. We know that transmission data rate is increased as the transmission power of UEs is increased that leads to energy efficiency enhancement. However, in the absent of perfect information of channel conditions, UEs consume higher circuit power to transmit data causing energy efficiency reduction. The proposed approach effectively adapts the transmission power to the true channel propagation condition results in reducing circuit power consumption. However, as shown, the proposed approach enhances data transmission rate and outperforms the existing schemes in terms of energy efficiency.

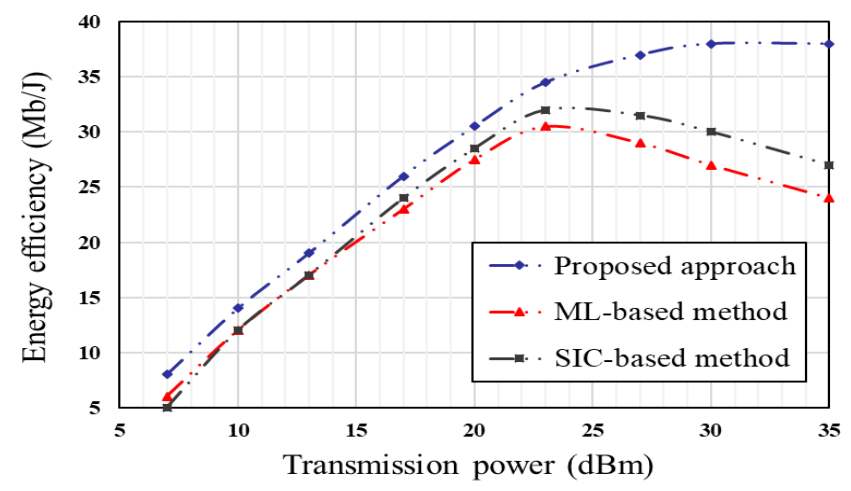

Fig. 1. Network energy efficiency for different transmission power

We investigate the effect of estimation error on the average power consumption for all existing schemes in Fig.2. The figure clearly shows that all methods are oriented towards higher and faster power consumption at higher estimation error. For instance, in the proposed approach, the average power consumption is approximately $3800 \mathrm{~W}$ for $\xi^{2}=0.01$ while it reaches over $4650 \mathrm{~W}$ at $\xi^{2}=0.1$. The total power consumption for ML-based scheme and SIC-based algorithm are $4950 \mathrm{~W}$ and $5215 \mathrm{~W}$ under $\xi^{2}=0.1$, respectively. Consequently, the average power consumption increases under larger error variance. The reason is that, in high level of accuracy, smart devices can reduce their transmission power since they adapt the transmission power with true channel characterization. We also study the impact of estimation error on the energy efficiency performance in Fig. 3.

Fig. 4 illustrates the data transmission rate under various transmission power for different values of error variances. The findings explicitly demonstrate that the data transmission rate is vulnerable to estimation error. For instance, the average data transmission rate of the proposed approach is almost $8.6 * 10^{9}$ bps at $\xi^{2}=0.01$ while it is about $5.6 * 10^{9}$ bps for larger error variance $\xi^{2}=0.1$. Thus, we can deduct that the higher estimation error results in lower data transmission rate. In fact, As the size of error estimation is increased, the average transmission rate degrades dramatically.

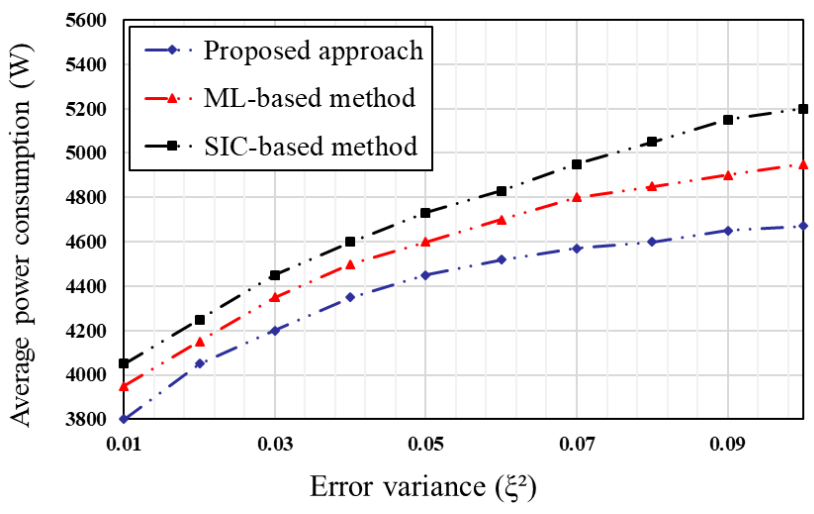

Fig. 2. Average power consumption for different $\xi^{2}$. 


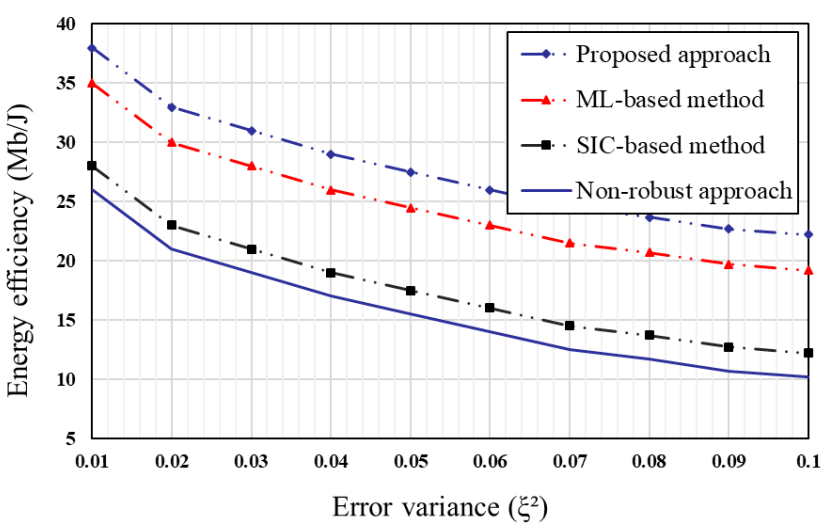

Fig. 3. Energy efficiency under different $\xi^{2}$.

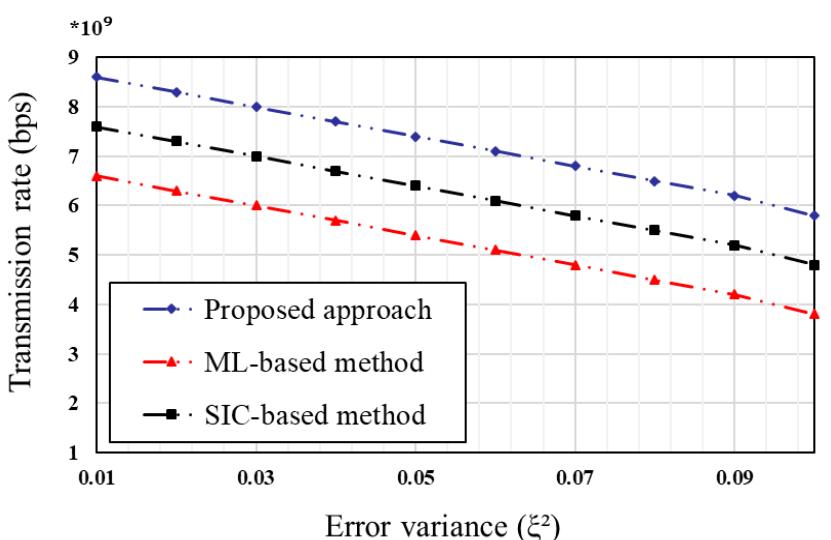

Fig. 4. Data transmission rate at different $\xi^{2}$.

Fig. 5 demonstrates the mean square error (MSE) performance of all the methods for comparison.

The graph confirms that the superiority of our approach compared with the baseline methods. It can be seen that approximately MSE $<0.27$ for our method whereas it is nearly 0.45 in SIC-based scheme and 0.37 in ML-based algorithm. Therefore, it is evident that the proposed approach provides high accuracy estimation that leads to lower power consumption and higher energy efficiency.

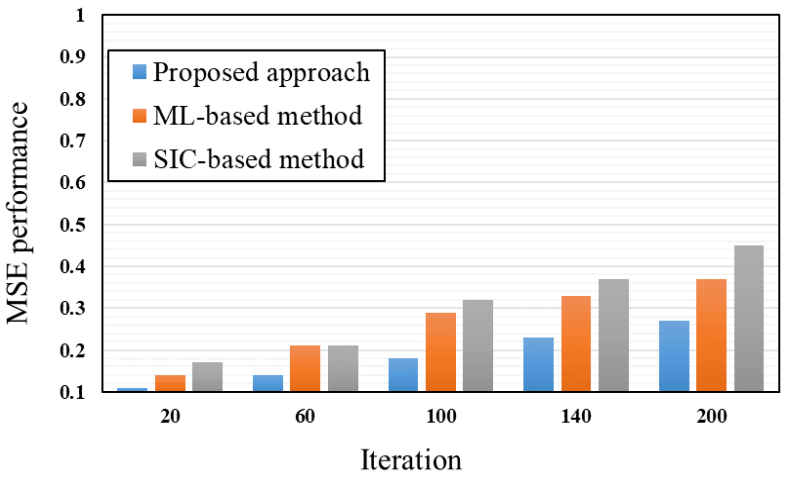

Fig. 5. MSE analysis for the existing methods under different iterations.

\section{CONCLUSION}

In this study, we proposed a robust energy-efficient data rate allocation method for a multiuser $6 \mathrm{G}$ system that is less sensitive to the channel uncertainty. We considered time-varying channel model since the performance of the rate allocation process extremely depends on the channel state. Due to the perfect CSI is not available in realistic scenarios, we used maximum likelihood estimator to obtain accurate estimation of channel characterizations. The proposed estimation approach obtained accurate channel coefficients for optimal rate allocation. We then established a distributed algorithm to adapt data transmission rate to true channel conditions and solve the optimization problem. Eventually, simulation findings revealed that the proposed method enhances the network energy efficiency and achieves higher data transmission rate comparing to the existing schemes. As a future work, we are planning to develop an antenna selection algorithm in order to switch off a number of receive antennas based on the channel conditions. Antenna selection methods emphasize activation of only a few number of antenna while keeping bit resolution on high. This reduces circuit power consumption and enhances energy efficiency in massive MIMO systems while the channel capacity remains the same. Also, we intend to design an efficient userantennas scheduling scheme as it provides higher data transmission rate and more energy saving for $6 \mathrm{G}$ communications with large number of users.

\section{REFERENCES}

[1] W. Saad, M. Bennis, and M. Chen, "A vision of 6G wireless systems: Applications, trends, technologies, and open research problems," IEEE Netw., vol. 34, no. 3, pp. 134-142, 2020.

[2] T. S. Rappaport et al., "Wireless communications and applications above $100 \mathrm{GHz}$ : Opportunities and challenges for 6G and beyond," IEEE Access, vol. 7, pp. 78729-78757, 2019.

[3] P. Yang et al., "6G wireless communications: Vision and potential techniques," IEEE Netw., vol. 33, no. 4, pp. 70-75, Jul. 2019.

[4] M. Giordani et al., "Toward 6G networks: Use cases and technologies," IEEE Commun. Mag., vol. 58, no. 3, Mar. 2020.

[5] H. T. Chen, A. J. Taylor, and N. Yu, "A review of meta-surfaces: Physics and applications," Reports on Progress in Physics, vol. 79, No. 7, 076401, 2016.

[6] Matthaiou, Michail, Okan Yurduseven, Hien Quoc Ngo, David MoralesJimenez, Simon L. Cotton, and Vincent F. Fusco. "The road to 6G: Ten physical layer challenges for communications engineers." IEEE Communications Magazine 59, no. 1 pp. 64-69, 2021.

[7] A. Fehske, J. Malmodin, G. Biczok, and G. Fettweis, "The global footprint of mobile communications: The ecological and economic perspective," IEEE Commun. Mag., pp. 55-62, 2011.

[8] Tang, Liangrui, and Hailin Hu. "Computation offloading and resource allocation for the Internet of Things in energy-constrained MEC-enabled HetNets." IEEE Access 8, 47509-47521, 2020.

[9] Sodhro, Ali Hassan, Noman Zahid, Lei Wang, Sandeep Pirbhulal, Yacine Ouzrout Ouzrout, Aicha Sekhari, Aloisio Vieira Lira Neto, Antonio Roberto L. De Macedo, and Victor Hugo C. De Albuquerque. "Towards ML-based Energy-Efficient Mechanism for 6G Enabled Industrial Network in Box Systems." IEEE Transactions on Industrial Informatics, 2020 .

[10] Sodhro, Ali Hassan, Sandeep Pirbhulal, Luo Zongwei, Khan Muhammad, and Noman Zahid. "Towards 6G Architecture for Energy Efficient Communication in IoT-Enabled Smart Automation Systems." IEEE Internet of Things Journal, 2020.

[11] Zakeri, Abolfazl, Ata Khalili, Mohammad Reza Javan, Nader Mokari, and Eduard Jorswieck. "Robust energy-efficient resource management, SIC 
ordering, and beamforming design for MC MISO-NOMA enabled 6G." IEEE Transactions on Signal Processing 69, pp. 2481-2498, 2021.

[12] A. Zappone, E. Bjornson, L. Sanguinetti, and E. Jorswieck, "Globally " optimal energy-efficient power control and receiver design in wireless networks," IEEE Trans. Signal Process., vol. 65, no. 11, pp. 2844-2859, 1 Jun. 2017.

[13] Pourkabirian, Azadeh, and Mohammad Hossein Anisi. "Robust Channel Estimation in Multiuser Downlink 5G Systems Under Channel Uncertainties." IEEE Transactions on Mobile Computing, 2021.

[14] Mukherjee, Amrit, Pratik Goswami, Mohammad Ayoub Khan, Li Manman, Lixia Yang, and Prashant Pillai. "Energy Efficient Resource Allocation strategy in Massive IoT for Industrial 6G Applications." IEEE Internet of Things Journal, 2020.

[15] Q. Shi, C. Peng, W. Xu, M. Hong, and Y. Cai, "Energy efficiency optimization for miso swipt systems with zero-forcing beamforming," IEEE Transactions on Signal Processing, vol. 4, no. 64, pp. 842-854, 2016.

[16] A. Pourkabirian, M. Dehghan Takht Fooladi, E. Zeinali, and A.M. Rahmani, "Dynamic resource allocation for OFDMA femtocell networks: a game-theoretic approach," Telecommunication Systems, vol. 69, no. 1, pp. 51-59, 2018.

[17] E. Björnson, J. Hoydis, and L. Sanguinetti, "Massive MIMO networks: Spectral, energy, and hardware efficiency," Foundations and Trends in Signal Processing, vol. 11, no. 3-4, pp. 154-655, 2017. 